International Journal of Advanced Chemistry, 8(1)(2020) $155-159$
International Journal of Advanced Chemistry
SPC
Website: www.sciencepubco.com/index.php/IJAC
Research paper

\title{
Formulation of Paint using Natural Pigment from Lawsonia Inermis Leaves
}

\author{
Z. Y. Abba ${ }^{1}$, S. M. Gumel ${ }^{2}$, A. A. Idris ${ }^{2}$, M. A. Ibrahim ${ }^{2}$ \\ ${ }^{1}$ Department of Chemical Science, Federal University of Kashere P.M.B 0182, Gombe, Nigeria \\ ${ }^{2}$ Department of Pure and Industrial Chemistry, Bayero University, P.M.B. 3011, Kano, Nigeria \\ *Corresponding author E-mail: zulyadainiyahuza@gmail.com
}

\begin{abstract}
The increased application of paint in coating industry for interior and exterior decoration and corrosion inhibition, has inspired research in paint formulations. However, due to the toxic effect of preparatory chemicals used in paints, and the restrictive environmental legislation, research efforts have been directed to a more green process. In this work colouring matter from Lawsonia inermis leaves were extracted using water and analyzed by TLC and FTIR spectroscopy. White emulsion paint was formulated using pigment volume concentration (PVC) of $0.07 \%$, the colouring matter extract was dispersed providing colour for the paint. The paint produced with the natural colourant was found to have similar properties with the paint produced using commercial pigment. Paint produced with aqueous extract faded under exposure to 5000 watts Tungsten Lamp after 48 hours but showed excellent fastness to rubbing. The extract induced excellent brownish colouration in the paint which maintained excellent, opacity, adhesion and good water resistance.
\end{abstract}

Keywords: Paint; Paint Formulation; Lawsonia Inermis Leaves; PVC; TLC; FTIR.

\section{Introduction}

An acceleration of the rate of scientific discovery had a growing impact on the development of paints from the eighteenth century to the present day. The quality of our environment is deteriorating day by day due to the threat impose by harmful chemical substances as a result of industrialization, in which largest cities reaching saturation points are unable to cope with the increasing pressure on their infrastructure (Lakherwal, 2014). Paints can be defined as a fluid, with viscosity, drying time and flowing properties dictated by formulation, normally consisting of a vehicle or binder, a pigment, a solvent or thinner and a drier which may be applied in relatively thin layers and which changes to a thin opaque continuous layer on surfaces (Nwakaudu and Oghome, 2012). Several types of paints have been classified, among which include solvent (water and oil) based paint and substrate (exterior, interior, automotive, marine and industrial) based paint (Adamu et al., 2014). Paints are used for decoration and also play a vital role in protecting major assets from the natural processes (Suma et al., 2009). The constituents generally used for the production of emulsion house paints include pigments, solvents, extenders, binders and additives (Abdulsalam and Yahaya, 2010). Due to perceived safety and physiological advantage of the natural colourants over synthetic ones, interest are being geared into search of new natural colourants and the verification of the safety of existing ones (Duangmal et al., 2004). They are also used for protecting surfaces against environmental effects like UV-radiation, chemical invasion and mechanical stresses (Oladipo et al., 2013). Due to increasing use of synthetic colourants which are mostly toxic as well as for environmental safety, it has become imperative to develop alternative source with similar properties (Omojola and Alabi, 2013). Poly (vinyl acetate) the widely used binder in paint formulation suffers poor hydrolytic stability especially under alkaline condition, poor weathering stability, poor wet adhesion and high-water sensitivity. It has only limited application in exterior coating because of the high $\mathrm{Tg}\left(30{ }^{\circ} \mathrm{C}\right)$ and minimum film forming temperature (MFT) $20^{\circ} \mathrm{C}$ making its film too hard and inflexible limiting its use in paint application (Suma et al., 2009).

Henna plant is a tall flowering tree standing about $5 \mathrm{~m}$ tall, native to tropical and subtropical regions of Africa, Asia and Northern nations and it's considered as harmless. The well-known compound is Lawsone occurred in the henna plant leaves. Phytochemists gave the compound the trivial name Lawsone due to its origin, the henna plant Lawsonia inermis. Lawsone is an intact glycosidase, able to split the glycosidic bond, when brought into contact with hot water. Therefore, Lawsone has been extracted by means of maceration, digestion and infusion (Mahkam et al, 2014).

There were number of works conducted by different researchers using locally available source materials to produce emulsion paint. Therefore, this present work focuses on formulation, characterization and application of an emulsion paint using poly (vinyl pyrrolidone) as binder and natural colouring matter from Lawsonia inermis leaves to provide colour for the paint. 


\section{Materials and Methods}

\subsection{Chemicals and Materials}

Dried Lawsonia inermis leaves was purchased from Rimi market, Kano State of Nigeria. Poly(vinylpyrrolidone) (Sigma Aldrich), Calgon; Sodium polymetaphosphate (PubChem: 24968) obtained from Clear Paint and Chemicals Company Limited, Technology Incubation Center, Kano, was used as water softener, Hydroxylethylcellulose (Ashland Specialty Chemical), was used for thickening purpose. Calcium carbonate (Specialty Minerals), was used as extender and Titanium dioxide (Winchems) was used for pigmenting. Ammonia (BHD), and biocide (Medimark Scientific Ltd) were purchased from Clear paints and used as preservatives. In order to prevent foaming, Anti-foaming agent was used. All chemicals and solvents used were of analytical grade and were used without further purification. The glass wares were washed with chromic acid and detergent, rinsed with distilled water and dried in oven before used.

\subsection{Extraction of Colouring Matter from Lawsonia Inermis Leaves}

Hot water extraction procedure was adopted (Saeed et. al., 2013) with little modification in the solid: solvent ratio. Here $100 \mathrm{~g}$ each of the plants was soaked in $1000 \mathrm{ml}$ distilled water and heated at $100{ }^{\circ} \mathrm{C}$ for $45 \mathrm{~min}$ under stirring. The system was then allowed to cool and filtered using a whatman filter paper to remove insoluble foreign particles. The filtrate was then concentrated using rotary evaporator from which the rotary flask was filled with the extract, and mounted in the rotor vapour, the machine was adjusted to insert the evaporating flask into water bath, which was set at $60{ }^{\circ} \mathrm{C}$. A vacuum pump was connected with the rotor vapour which exert the required pressure $72 \mathrm{~atm}$. A recycling chiller was attached with the condenser which condense the vapour and collected via receiving flask. The samples was further dried using freeze drying machine and kept air tight container for further analysis.

\subsection{Characterization}

\subsubsection{Determination of Extract Percentage Yield}

The yield of the plant extract were determined as reported elsewhere (Anokwuru et al., 2011) using the formula576i9c

$\frac{\mathrm{W}_{2-} \mathrm{W}_{1}}{\mathrm{~W}_{\mathrm{o}}} \times 100 \%$

Where: $\mathrm{W}_{2}$ is the weight of the extract and the container,

$\mathrm{W}_{1}$ the weight of the container alone and

$\mathrm{W}_{0}$ is the weight of the initial dried sample

\subsubsection{FTIR Studies}

The chemical functional groups of the extract were revealed using the Fourier transformed infra-red spectroscopic technique. Sample was directly placed on a cuvette and the transmittance spectrum of both the extract and the formulated paint were recorded using Shimadzu FTIR model No. 8400S.

\subsubsection{TLC Separation and Analysis}

The various components of the extract were separated using Thin Layer Liquid Chromatography. An aliquot of the extract was dissolved in ethanol to produce a very dilute (about 1\%) solution. A small amount of the solution was transferred on one end of a TLC plate which quickly evaporates leaving behind small spot of the extracts on the TLC plate. The spotted end was placed into a beaker containing four different solvents (N-Hexane, ethyl acetate, chloroform and methanol), which then travels up the plate from the original spot by capillary action. The spots travelled almost to the top of the plate. Three different colours were observed; green, light and dark brown.

\subsection{Paint Formulation}

The paint formulation was initiated by first dissolving $2.5 \mathrm{~g}$ of sodium polymetaphosphate (Calgon) in $750 \mathrm{ml}$ of distilled water. To this solution, $37.5 \mathrm{~g}$ of $\left(\mathrm{TiO}_{2}\right)$ was added and shaked vigorously in a plastic container. For the purpose of gloss $325 \mathrm{~g} \mathrm{CaCO} 3$ was added to the mixture and stirred for 20 minutes. A specified amount of Poly (vinylpyrrolidone) was added as binder under stirring condition. For thickening purpose $5 \mathrm{~g}$ of Hydroxylethylcellulose was added. Finally, the preserving agents were added in equal proportion.

\subsection{Paint Property Assessments}

\subsubsection{Drying Time}

The drying time of the formulated sample was determined. The paint sample was applied on a substrate (interlocks) and drying time was determined by exposure to air and recorded.

\subsubsection{Weathering Test}

Weather ability (i.e. outdoor durability) of emulsion paint coating like other coating systems is expressed as change in gloss, opacity, colour retention, vertical wall sagging, rubbing, resistance to staining and light fastness test. All these processes are influenced by nature, like solar radiation, temperature, moisture, chemical pollutants, oxygen, and microorganisms. The sample of the formulated paint pattern was exposed in an open air during the weathering test for $240 \mathrm{hrs}$. 


\subsubsection{Fastness Tests}

\section{Determination of Light Fastness}

The test was carried out on the painted samples with use of a 500 watts fading lamp. The procedure involved aligning both the eight blue standards and the painted samples, until the last specimen faded. The rating of the painted samples was carried out to assess the degree of fading.

\section{Determination of Rubbing Fastness}

Rubbing fastness is the resistance of the dyed material or a paint substrate to rubbing. In the present research the test was conducted on the painted interlock blocks both in dry and wet condition.

\section{Results and Discussions}

\subsection{Percentage Yield of the Plants Extract}

The initial weight of the container alone $\mathrm{W}_{1}=34.1274 \mathrm{~g}$

Weight of the initial dried sample $\mathrm{W}_{0}=100 \mathrm{~g}$

Weight of the extract + weight of the container $\mathrm{W}_{2}=27.6742+34.1274=61.8016 \mathrm{~g}$

Therefore \% yield of the Lawsonia inermis plant $=\frac{61.8016-34.1274}{100 \mathrm{~g}} \times 100$

$=27.6742 \%$

\subsection{Determination of Pigment Volume Concentration (PVC)}

PVC for $37.5 \mathrm{~g}$ of pigment: The pigment volume concentrations (PVC) for the formulated paint was determined. The PVC for $37.5 \mathrm{~g}$ of pigment was found as represented from the table below. From table 1, emulsion paint was prepared at the PVC of 0.07 (7\%) CPVC. CPVC which is the stage where air occupying the interstitial spaces of the pigment has been displaced by binder. Any increase in pigment concentration above CPVC level lead to voids in the paint film with a consequent increase in permeability, and below CPVC leads to the separation of pigment particles from each other. The CPVC increases with increasing amount of dispersing agent indicating that the close packing of pigment particles is intimately associated with degree of dispersion. When the pigment dispersed on the formulated paint is below the critical pigment volume concentration, the paint may become rucked in patches as a result of efflorescence (Udeozo et al., 2013).

Table 1: PVC (Pigment Volume Concentration) for $37.5 \mathrm{~g}$ of $\mathrm{TiO}_{2}$ Pigment

\begin{tabular}{llll}
\hline Component & Weight & Density & Volume \\
\hline Pigment & 37.5 & 4.23 & 8.86 \\
PVP & 125 & 1.2 & 104.16 \\
\hline
\end{tabular}

$\mathrm{PVC}=\frac{\mathrm{V}_{\mathrm{P}}}{\mathrm{V}_{\mathrm{p}}+\mathrm{V}_{\mathrm{b}}}$

Where $V_{P}$ and $V_{b}$ are the Pigment volume and volume of the binder respectively.

$$
\begin{aligned}
& \mathrm{PVC}=\frac{8.86}{8.86+104.16}=\frac{8.86}{113.02} \\
& =0.07 \\
& \mathrm{CPVC}=0.07 \times 100 \\
& \mathrm{CPVC}=7 \%
\end{aligned}
$$

\subsection{Thin Layer Chromatographic Technique of the Plant Extracts}

The Thin Layer Chromatographic Technique (TLC) of the plant extracts was conducted. The number of components in the mixture were traced based on the colours appear on the TLC plate by observing their appearance, where the hues obtained due to stationary and mobile phases were clearly seen.

The results presented here were obtained from Thin Layer Chromatography (TLC), which is a method for analyzing mixtures by separating the compounds in a mixture. TLC can be used to help determine the number of components in a mixture, the identity and purity of compounds. The number of components in the mixture were traced based on the colours appear on the TLC plate by observing the appearance of a product or the disappearance of a reactant. It can also be used to monitor the progress of a reaction. Thin Layer Chromatography (TLC) is a sensitive technique from which microgram $(0.000001 \mathrm{~g})$ quantities can be analysed and it takes little time for an analysis (about 5-10 minutes). Figure 1 present the TLC of Lawsonia inermis where the colours obtained due to stationary and mobile phases were clearly seen. 


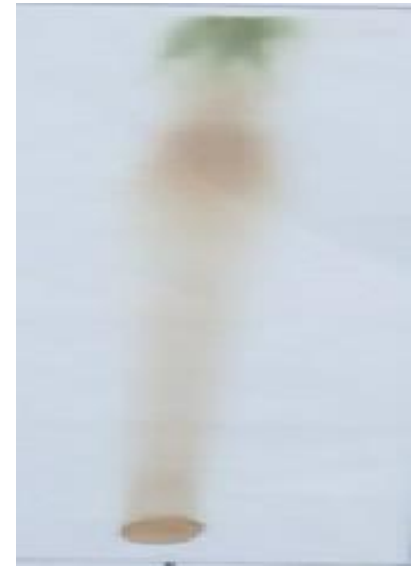

Fig. 1: TLC Image of L. Inermis Leaves Extracts Showing Different Components.

The figure shows three (3) colours from Lawsonia inermis namely; green, light and dark brown. When the extracts were mixed with polyvinylpyrrolidone as a binder and other additives in emulsion paint formulation, brown colour was observed from Lawsonia inermis, while the remaining colours were not seen. It was also observed that there is good compatibility between the extracts and the binder during paint formulation.

\subsection{FTIR Spectral Characterization of the Plant Extracts and the Formulated Paints}

The results presented here were based on the use of Fourier Transform Infra-red Spectroscopy (FT-IR) Machine from which the examination of the spectra reveals the presence of some functional groups found on Lawsonia inermis Extract.

Table 2 also revealed the FTIR spectrum of Lawsonia inermis leaves extract. Peaks observed at $3402 \mathrm{~cm}^{-1}$ was due to O-H stretching while that at $2924 \mathrm{~cm}^{-1}$ was attributed to $\mathrm{C}-\mathrm{H}$ stretching $\left(\mathrm{sp}^{3}\right)$. Other peaks at 1635 and $1141 \mathrm{~cm}^{-1}$ were due to the presence of $\mathrm{C}=\mathrm{C}$ confirming their presence from the structure of the active compound of henna leaves. FTIR Spectrum of the white emulsion paint was represented in table 3. The characteristics peaks found at 3448 and $2931 \mathrm{~cm}^{-1}$ were due to O-H stretching and C-H stretching ( $\mathrm{sp}^{3}$ ). All the peaks were present even after paint formulation confirming that there was no chemical reaction during the formulation.

Lastly, result from Table 3 highlight the FTIR of emulsion paint formulated with Lawsonia inermis leaves extract as colour impacting agent. Characteristic peak at $3410 \mathrm{~cm}^{-1}$ was indicating $\mathrm{O}-\mathrm{H}$ stretching, a sharp peak at $2931 \mathrm{~cm}^{-1}$ was due to the presence of C-H stretching $\left(\mathrm{sp}^{3}\right)$ and peak at $1419 \mathrm{~cm}^{-1}$ band was attributed to $\mathrm{C}-\mathrm{C}$ from the aromatic ring of benzene of Lawsonia inermis leaves extract.

Table 2: Summary of FTIR Results of Extracts from Lawsonia Inermis Leaves

\begin{tabular}{lll}
\hline Peaks & Lawsonia inermis & Functional Group Assignment \\
\hline 1 & 3402 & O-H \\
2 & 2924 & C-H $\left(\mathrm{sp}^{3}\right)$ \\
3 & 1635 & $\mathrm{C}=\mathrm{C}$ \\
4 & 1141 & $\mathrm{C}=\mathrm{C}$ \\
\hline
\end{tabular}

Table 3: Summary of FTIR Results of Emulsion Paint Samples

\begin{tabular}{lllll}
\hline Peaks & Emulsion paint & Functional group Assignment & Emulsion paint with Lawsonia inermis & Functional Group Assignment \\
\hline 1 & 3448 & O-H & 3410 & O-H \\
2 & 2931 & C-H $\left(\mathrm{sp}^{3}\right)$ & 2931 & C-H ( $\left.\mathrm{sp}^{3}\right)$ \\
3 & & & 1419 & C-C (Ar) \\
\hline
\end{tabular}

\subsection{Application of Formulated Paint}

The performance characteristics of the paints were determined in terms of drying time, opacity, adhesion, resistance to rubbing, pigment volume concentration (PVC), light fastness and hue observed where recorded in Table 4 . The paint formulated coloured with the extract were applied on interlock blocks and dried at room temperature.

\subsubsection{Hue Observed}

Three different hues on the TLC of Lawsonia inermis namely; green, light and dark brown, respectively were observed.

\subsubsection{Drying Time}

Drying or curing time of the paints on interlock blocks was observed and recorded. From the result obtained, white emulsion paint dry fast at 8mins, while emulsion paint coloured with Lawsonia inermis extracts dries at $15 \mathrm{mins}$. This indicate that Lawsonia inermis extract delays the drying of the paint to some extent due to oily nature of the extract.

\subsubsection{Adhesion}

The paints samples were applied on interlock blocks and adhesion of the paint on the substrate was observed visually from which all the paint samples; White emulsion paint and paint coloured with the extracts from Lawsonia inermis shows excellent adhesion. 


\subsubsection{Opacity (Hiding Power)}

From the result obtained opacity (hiding power) of the paint was measured by visual assessment; five different observers rated the degree of opacity of the paint formulations. From the formulations of the paints white emulsion paint shows excellent opacity while emulsion paint coloured with Lawsonia inermis extracts indicate good hiding power when applied on the interlock blocks.

\subsubsection{Light Fastness Property}

The light fastness test was carried out on the paint samples using cupboard paper, from which the formulated paint coloured with Lawsonia inermis extract having grade 4 comparing with the 8 blue wool standard.

Light Fastness Rating Key: 1= Poor; 2= slightly poor; 3= Moderate; 4= Fair; 5= Good; 6=Very good; 7= Outstanding; 8= Excellent

\subsubsection{Rubbing Fastness Property}

The obtained results deduced that both at dry and wet conditions the paint samples tested for staining white cotton fabric have good resistance to rubbing, from which the formulated paint with Lawsonia inermis had excellent fastness both in dry and wet conditions with rating 5 .

Rubbing Fastness Rating Key: 1= Poor; 2= Fair; 3= Good; 4= Very good; 5= Excellent.

Table 4: Paint Property Assessment

\begin{tabular}{lllllll}
\hline Paint Samples & Hue & Adhesion & Opacity & Drying Time (min) & Water Resistance & Light fastness \\
\hline White Emulsion Paint & White & Excellent & Excellent & 8 & Excellent & Gobing fastness \\
With Lawsonia inermis & Brown & Excellent & Excellent & 15 & Good & 4 \\
\hline
\end{tabular}

\section{Conclusion}

In this research, paint was successfully formulated by the use of Lawsonia inermis leaves extract and has also been explored to provide colour in paint formulations, percentage yield of the plant extract was calculated. Thin layer chromatographic technique (TLC) revealed different colours from the TLC plates under observation. The bands in the FTIR spectra of the plant extract and the formulated paints reveals same functional groups indicating that there was no chemical reaction taking place during paint formulation. From the result obtained, it can be concluded that extracts from the plant have the same effect of impacting colour with the toxic inorganic pigments used in paint industry and can also be used to replace them and turn out to be of great contribution in commercial sectors. As such the paints formulated can be considered a satisfactory article of commerce.

\section{References}

[1] Abdulsalam, S. and Yahaya, Y. U. (2010), "Effectiveness of Gum Arabic as a Binder in Emulsion House Paint", Global Journal of Engineering Research, 10(2): 83-89.

[2] Adamu, A. K., Yakubu, M. K. and Sunmonu, O. K. (2014). Characterization of Emulsion Paints Formulated using Reactive - Dyed Starch as a Pigment. International Conference on Biological, Chemical and Environmental Sciences (BCES), 1(1): 2320-4087.

[3] Anokwuru, C. P, Anyasor, G. N, Ajibaye, O, Fakoya, O and Okebugwu P. (2011). "Effect of Extraction Solvents on Phenolic, Flavonoid and Antioxidant Activities of Three Nigerian Medicinal Plants" Nature and Science; 9(7): 53-61.

[4] Duangmal, K., Saicheua, B. and Sueeprasan, S. (2004). Roselle Anthocyanins as a Natural Food Colourant and Improvement of Its Colour Stability. Proceedings of the AIC Colour and Paints, Interim Meeting of the International Colour Association, pp: 155-158.

[5] Lakherwal, D. (2014). "Adsorption of Heavy Metals". International Journal of Environmental research and Development, 4(1): 41-48.

[6] Mahkam, M., Mehdi, N. and Kafshboran, H. R. (2014). "Isolation, Identification and Characterization of Lawsone from Henna Leaves Powder with Soxhlet Technique", Iranian Chemical Communication, 2(4): 34-38.

[7] Nwakaudu, M. S. and Oghome, P. (2012). "Production of Paint from Locally Sourced Raw Materials" International Journal of Science and Engineering Investigations, 1(4): 2251-8843.

[8] Oladipo, 1., Gabriel, O., Ighodalo, C. Eromosele, Olujinmi, M. and Folarin. (2013). "Formation and Characterization of Paint Based on Alkyd Resin Derivative of Ximenia americana (Wild Olive) Seed Oil" Environment and Natural Resources Research, 3(3): 52-62. https://doi.org/10.5539/enrr.v3n3p52.

[9] Omojola, M., O. and Alabi, F. M. (2013). "Potentials of Nigerian Calcined Kaolin as Paint Pigment”. Raw Materials Research and Development Council PMB 232, Garki Abuja, Nigeria, 7(12): 410-417.

[10] Suma, K. K., Sinto, J. and Rani J. (2009). "Paint Formulation Using Water Based Binder and Property Studies". Macromol Symp, $277(8): 144-151$. https://doi.org/10.1002/masy.200950318.

[11] Udeozo, I. P., Umedum, N. L., Okoye, N. H. and Kelle, I.H. (2013). Formulation of Glossy Emulsion Paint, International Journal of Science and Technology, 13(1): 822-828. 